\title{
Tolerancia a diferentes factores antinutritivos en lechones alimentados con guisantes de invierno (Pisum sativum) y alberjón (Vicia narbonensis) en la fase estárter
}

\author{
E. Gómez-Izquierdo 1, ${ }^{*}$, E. de Mercado ${ }^{1}$, J. Gómez-Fernández ${ }^{1}$, C. Tomás ${ }^{1}$, \\ E. Guillamón ${ }^{2}$ A. Varela 3 , M. Muzquiz ${ }^{3}$, M.M. Pedrosa ${ }^{3}$, \\ P. López-Nuez ${ }^{4}$ y M.A. Latorre ${ }^{5}$
}

1 Centro de Pruebas de Porcino del Instituto Tecnológico Agrario de Castilla y León. Consejería de Agricultura y Ganadería. Ctra. Riaza a Toro s/n, Hontalbilla, 40353, Segovia, España

2 Centro para la Calidad de los Alimentos, INIA, Campus Universitario Duques de Soria, 42004, Soria, España

3 Departamento de Tecnología de los Alimentos, INIA, Ctra. A Coruña km 7,5, 28040, Madrid, España

4 Copiso Soria Sociedad Cooperativa, Av. de Valladolid 105, 42005, Soria, España

5 Departamento de Producción Animal y Ciencia de los Alimentos, Facultad de Veterinaria, Universidad de Zaragoza, Avda. Miguel Servet, 177, 50013, Zaragoza, España

\section{Resumen}

Se realizaron dos ensayos para evaluar el efecto de dietas con diferentes niveles de factores antinutritivos: inhibidores de tripsina y quimotripsina (UTI, UQI respectivamente, como unidades) de guisantes de invierno y $\gamma$ Glutamyl-S-Ethenyl-Cysteine de alberjón, en el rendimiento de lechones durante el periodo estárter. En cada uno se utilizaron 192 machos castrados, híbridos Duroc x (Large-White x Landrace), con 11,23 y 11,55 kg de peso medio respectivamente, e igual diseño: cuatro tratamientos y ocho réplicas cada una de seis lechones. Los tratamientos del Ensayo 1 fueron: Control: harina de soja 47 y soja extrusionada (0,97 UTI-1,94 UQI/mg de pienso); P-CAR: soja extrusionada y guisante Cartouche (1,62 UTI3,16 UQI/mg de pienso); P-ICE: soja extrusionada y guisante Iceberg (3,09 UTI-4,95 UQI/mg de pienso); P-LUN: soja extrusionada y guisante Luna (3,19 UTI-5,56 UQI/mg de pienso). Los lechones del tratamiento P-CAR crecieron significativamente más que el resto, con igual consumo y conversión. No hubo diferencias entre Control, P-ICE y P-LUN. El pienso del Ensayo 2 incluía diferentes niveles de alberjón: 0\%, $5 \%, 15 \%$ y $25 \%$, y en consecuencia de $\gamma$ Glutamyl-S-Ethenyl-Cysteine ( $1,52 \%$ del grano). Los lechones del tratamiento $5 \%$ comieron y crecieron más, con igual conversión que los del tratamiento $0 \%$. El rendimiento empeoró con porcentajes del $15 \%$ o mayores. Se concluye que lechones de 40 a 61 días de vida mantienen o mejoran el rendimiento con la ingesta de inhibidores de proteasas que exceden 3,28 y 2,86 veces, UTI y UQI respectivamente, los de una dieta de soja, y que la inclusión de $5 \%$ de alberjón en el pienso aumenta el consumo y el crecimiento.

Palabras clave: Proteaginosas, factores bioactivos, alimentación de lechones.

\section{Abstract \\ Tolerance to various anti-nutritional factors in young pigs fed on winter peas (Pisum sativum) and narbon vetch (Vicia narbonensis) in the starter phase}

Two trials were conducted to evaluate the effect of diets with different levels of anti-nutritional factors: trypsin (TIU) and chymotrypsin (CIU) inhibitors units of peas and $\gamma$ Glutamyl-S-Ethenyl-Cysteine (GEC) of nar-

\footnotetext{
* Autor para correspondencia: gomizqem@itacyl.es https://doi.org/10.12706/itea.2018.015
} 
bon vetch, in productive performance of pigs from 40 to 61 days of age. 192 barrows were used in each test, Duroc hybrid x (Large White x Landrace), with 11.23 and $11.55 \mathrm{~kg}$ body weight respectively, and the same experimental design: four treatments and eight replicates with six piglets in each case. In trial 1 treatments were: Control: soybean meal 47 and soybean extruded (0.97 TIU-1.94 CIU/mg feed); P-CAR: soybean extruded and pea Cartouche (1.62 TIU-3.16 ClU/mg feed); P-ICE: soybean extruded and pea Iceberg (3.09 TIU-4.95 CIU/mg feed); P-LUN: soybean extruded and pea Luna (3.19 TIU-5.56 CIU/mg feed). The piglets of P-CAR treatment grew significantly faster than the others, with the same intake and feed conversion. No significant differences between Control, P-ICE and P-LUN. In trial 2 the feedstuff had different levels of narbon vetch: $0 \%, 5 \%, 15 \%$ and $25 \%$, and consequently of $\gamma$ Glutamyl-S-Ethenyl-Cysteine $(1.52 \%$ of the grain). The piglets that consumed the treatment of $5 \%$ had higher intake and growth, and the same conversion that piglets feeding with the treatment of $0 \%$. The performance worsened significantly with increasing $15 \%$. We conclude that piglets in starter period maintain or improve the performance with intake of proteases inhibitors in feedstuff that exceed 3.28 (TIU) and 2.86 (CIU) times those of a control diet, and that the inclusion of $5 \%$ of narbon vetch in feedstuff increases feed intake and growth.

Keywords: Protein crops, biologically active factors, piglets feeding.

\section{Introducción}

Los factores antinutritivos (FAN) de las legumbres son el principal factor limitante para su inclusión en altos porcentajes en piensos de cerdos. Los guisantes (Pisum sativum) con un perfil de nutrientes de gran interés, sobre todo por el contenido de proteína (PB), contienen taninos, saponinas, glucósidos, oligosacáridos, lectinas, fitatos, inhibidores de amilasas e inhibidores de proteasas (IP) (Muzquiz, 2012). De todos ellos, los IP son los más problemáticos y pueden afectar al valor nutricional de los piensos. De hecho se han asociado con un empeoramiento de la actividad de enzimas proteolíticas causando una reducción en la digestibilidad proteica (Price et al., 1985; Guillamón et al., 2012). El resultado es un rendimiento menor, más acusado en animales jóvenes (Bengala-Freire et al., 1989; Mateos et al., 2008).

Los IP pueden ser inhibidores de tripsina (IT) o de quimotripsina (IQ) (UTI, UQI respectivamente, como unidades). El rango de contenido de IT en los guisantes es amplio, de 0,8 a $17,7 \mathrm{UTI} / \mathrm{mg}$ materia seca, dependiendo de las variedades y de las técnicas de cultivo (Grosjean et al., 2000). Generalmente, en los guisantes la cantidad de IQ es mayor que la de IT, aunque estos últimos son los que se utilizan como marcadores de la actividad inhibidora (Griffiths, 1984). Algunos tratamientos térmicos, como la extrusión (extrusión en húmedo) inactivan los IP y aumentan la biodisponibilidad de las proteínas (Adamidou et al., 2011), pero son caros y pueden afectar negativamente la digestibilidad de la lisina debido a la alta temperatura utilizada (Crevieu-Gabriel, 1999). Además de la variedad y las condiciones de crecimiento, debería tenerse en cuenta que las técnicas de labranza (siembra directa o de dos fases) pueden implicar reducciones del 27 a $44 \%$ de inhibidores de tripsina sin cambiar los nutrientes, hecho importante en el caso de las proteínas (Pisulewska y Pisulewski, 2000; González-García, 2001). Los alberjones (Vicia narbonensis) (ALB) son originarios de la cuenca mediterránea. Es un cultivo muy resistente a condiciones desfavorables del clima y suelo, así como a las patologías y plagas más comunes de las legumbres (Arias et al., 2004). Estas características, junto con su considerable rendimiento, que puede superar los $4.000 \mathrm{~kg} / \mathrm{ha}$, y su alta capacidad de mejora genética, hace de ellos una leguminosa de gran interés (García et al., 2006). Su composición nutricional, con una elevada proporción de PB y amino- 
ácidos (AA) azufrados, no se corresponde con las posibilidades prácticas de inclusión en piensos debido a la presencia del dipéptido $\gamma$ Glutamyl-S-Etenyl-Cysteine (GEC), el principal FAN de esta leguminosa, que es resistente al calor y a la presión, aunque sensible a soluciones ácidas o básicas (Enneking 1995a; Enneking et al., 1998; Arias et al., 2004). La limitada información disponible sugiere que, dependiendo de la variedad de ALB, el GEC varía de 0,4 a 3,77\% de la semilla (Castleman, 2000) y causa una disminución significativa en el consumo de alimento al transmitir un sabor azufrado a la dieta que empeora su palatabilidad (Enneking, 1995b).

Sin embargo, el contenido de GEC, al igual que el resto de FAN, es necesario para la defensa de las plantas y su adaptación al medio. Proporciona un sabor desagradable a los roedores, aves, conejos, etc. y su eliminación haría a las plantas más sensibles a las plagas, aumentando el tratamiento con fitosanitarios de los cultivos $y$, por lo tanto, encareciendo los piensos y contaminando el medio ambiente (Tate y Enneking, 2006).

Para estudiar el efecto productivo de los FAN de ambas leguminosas se realizaron dos ensayos con lechones durante el período de estárter (40 a 61 días de edad). En el Ensayo 1 se valoró la repercusión sobre el rendimiento del nivel de IP (IT, IQ) en cuatro dietas: dieta Control con harina de soja y soja extrusionada (SE) y tres dietas con diferentes variedades de guisantes de invierno, guisante Cartouche (CAR), guisante Iceberg (ICE) y guisante Luna (LUN), que aumentaron los niveles de IP, aunque no sustituyeron completamente a la soja. Se tuvo en cuenta que las variaciones en la composición del almidón (relación amilosa/amilopectina) no suponen una variación significativa en producción, como indican Doti et al. (2014).

En el Ensayo 2 se estudió, igualmente, el efecto sobre el rendimiento productivo de distintos porcentajes de ALB en cuatro dietas: Control con $0 \%, 5 \%, 15 \%$ y $25 \%$.

\section{Material y métodos}

\section{Animales e instalaciones experimentales}

Todos los procedimientos empleados en el ensayo cumplieron la normativa sobre protección de los animales utilizados para experimentación y otros fines científicos reflejada en el Boletín Oficial del Estado (BOE, 2013).

Se emplearon 384 lechones, 192 en cada ensayo, de 40 días de edad, híbridos Duroc $x$ (Landrace $x$ Large white), machos (castrados a los $5 \pm 3$ días de edad), procedentes de una granja comercial situada en San Pedro Manrique (Soria, España). Ambos ensayos se desarrollaron en la fase estárter y duraron 21 días. Al comienzo de los mismos, todos los cerdos fueron identificados y pesados individualmente, asignándose a los diferentes tratamientos experimentales en función del peso inicial: Ensayo 1: 11,23 $\pm 1,12 \mathrm{~kg}$; Ensayo 2: 11,55 \pm $0,84 \mathrm{~kg}$. Los dos ensayos se llevaron a cabo en las instalaciones de transición del Centro de Pruebas de Porcino del Instituto Tecnológico Agrario (ITACyL, Consejería de Agricultura y Ganadería de la Junta de Castilla y León), localizado en Hontalbilla (Segovia, España).

Los lechones se alojaron en cuatro salas de ocho departamentos, con 6 cerdos en cada uno (dos departamentos de cada tratamientos por sala; $100 \%$ rejilla; $0,68 \mathrm{~m}^{2} /$ cerdo). Cada departamento dispuso de un comedero con seis bocas y un bebedero de cazoleta. La temperatura ambiental se redujo de $27 \pm 2^{\circ} \mathrm{C}$ a $22,5 \pm 2^{\circ} \mathrm{C}\left(-1,5^{\circ} \mathrm{C} /\right.$ semana $)$ de acuerdo a las necesidades de los lechones.

\section{Dietas experimentales}

Los piensos fueron fabricados en COPISO Soria Sociedad Cooperativa (Soria), y se formularon de acuerdo con las tablas de FEDNA (2010) para cumplir o exceder los requerimientos nutricionales en cerdos de esa edad (FEDNA, 2013). Se administraron ad libitum en forma de gránulo de $2,5 \mathrm{~mm}$. La composición química analizada de los guisantes y ALB utilizados se muestran en la Tabla 1. 
Tabla 1. Composición química de las fuentes proteicas empleadas en el experimento: harina de soja, soja extrusionada, guisantes (Cartouche, Iceberg y Luna) y alberjón (en \% de materia fresca a no ser que ser indique lo contrario)

Table 1. Chemical composition of protein sources used in the trial: soybean meal, soybean extruded, peas (Cartouche, Iceberg and Luna) and narbon vecht (\%, as-fed basis unless otherwise indicated)

\begin{tabular}{|c|c|c|c|c|c|c|}
\hline & $\mathrm{HS}^{1}$ & $\mathrm{SE}^{2}$ & Cartouche & Iceberg & Luna & Alberjón \\
\hline \multicolumn{7}{|l|}{ Nutrientes } \\
\hline Materia seca & 88,00 & 89,90 & 89,95 & 90,45 & 90,01 & 88,40 \\
\hline Proteína bruta & 47,00 & 36,80 & 21,10 & 23,20 & 23,30 & 26,48 \\
\hline Lisina & 2,88 & 2,25 & 1,48 & 1,62 & 1,63 & 1,77 \\
\hline Metionina + Cistina & 1,38 & 1,07 & 0,51 & 0,56 & 0,56 & 0,74 \\
\hline Treonina & 1,85 & 1,46 & 0,80 & 0,88 & 0,89 & 0,97 \\
\hline Triptófano & 0,63 & 0,49 & 0,19 & 0,21 & 0,21 & 0,22 \\
\hline Isoleucina & 2,13 & 1,67 & 0,97 & 0,89 & 0,99 & 1,06 \\
\hline Leucina & & & 1,55 & 1,44 & 1,50 & 1,66 \\
\hline Valina & 2,27 & 1,77 & 1,20 & 0,96 & 1,19 & 1,12 \\
\hline Fibra bruta & 4,10 & 6,10 & 5,20 & 5,70 & 6,36 & 8,20 \\
\hline Grasa bruta & 1,90 & 19,20 & 1,48 & 1,15 & 1,15 & 1,55 \\
\hline Cenizas & 6,20 & 4,80 & 2,47 & 2,74 & 2,48 & 3,49 \\
\hline Almidón & 0,50 & & 43,36 & 40,88 & 43,56 & 32,25 \\
\hline Amilosa $^{3}$ & & & 35,17 & 34,46 & 36,36 & 25,6 \\
\hline Amilopectina $^{3}$ & & & 64,83 & 65,54 & 63,64 & 74,4 \\
\hline \multicolumn{7}{|l|}{ Factores antinutricionales } \\
\hline $\mathrm{UTI}^{4}$ & 0,61 & 2,36 & 9,87 & 5,75 & 12,55 & \\
\hline UQI $^{5}$ & 3,56 & 4,65 & 10,16 & 8,62 & 15,75 & \\
\hline AITP6 & & & 46,78 & 24,78 & 53,86 & \\
\hline $\mathrm{GEC}^{7}$ & & & & & & 1,52 \\
\hline Inositol fosfatos mg/g & 15,54 & 10,22 & 6,43 & 6,43 & 4,46 & 5,84 \\
\hline Compuestos fenólicos mg/g & 13,59 & 10,57 & 2,02 & 4,19 & 2,85 & 3,83 \\
\hline$\alpha$-galactósidos ${ }^{8} \mathrm{mg} / \mathrm{g}$ & 57,53 & 47,23 & 59,29 & 59,55 & 71,99 & 48,51 \\
\hline Lectinas $(\mathrm{HU})^{9}$ & 0,16 & 0,08 & 0,24 & 0,32 & 0,63 & nd \\
\hline
\end{tabular}

${ }^{1}$ Harina de soja (nutrientes según FEDNA, 2010). ${ }^{2}$ Soja extrusionada (nutrientes según FEDNA, 2010). ${ }^{3}$ Según $\%$ de almidón. ${ }^{4}$ Unidades de tripsina inhibida/mg. ${ }^{5}$ Unidades de quimotripsina inhibida/mg. ${ }^{6} \mathrm{Ac}-$ tividad inhibidora de tripsina por unidad de proteína bruta (100/PB\%*UTI). ${ }^{7} \gamma$ Glutamyl-S-Ethenyl-Cysteine. ${ }^{8}$ Rafinosa, Estaquiosa, Ciceritol, Verbascosa. ${ }^{9}$ Unidades de hemoaglutinación (nd: no detectado). 
En el Ensayo 1 se testaron 4 tratamientos experimentales, que se correspondían con tres dietas con diferentes variedades de guisantes de invierno: P-CAR, guisante Cartouche; PICE, guisante Iceberg; P-LUN, guisante luna, y una dieta Control que contenía harina de soja y soja extrusionada como fuentes de proteínas principales (Tabla 2). El nivel de inclusión de guisantes dependió del contenido de IP de cada variedad, de modo que la cantidad de inhibidores se duplicó o triplicó en relación con la presente en la dieta Control. Dicho nivel se estableció de acuerdo con la actividad inhibidora de tripsina por unidad de proteína bruta (AITP $=100 / \mathrm{PB} \%$ *UTI) $(\mathrm{Ta}$ bla 1), factor que tienen en cuenta las ecuaciones de predicción de Grosjean et al. (2000). De esta manera se determinó la digestibilidad ileal estandarizada de la PB $(-0,1975$. AITP + 84,72), Lisina $(-0,1617 \cdot$ AITP + 87,84), Metionina $(-0,2630 \cdot$ AITP $+86,46)$, Cistina $(-0,2029 \cdot$ AITP $+78,55)$, Treonina $(-0,2171$. AITP + 82,61) y Triptófano $(-0,3536 \cdot$ AITP + $80,44)$, y se facilitó la formulación de los piensos con guisantes. A pesar de ello, el nivel de IT y de IQ fue más alto en las dietas que contenían guisante que en la dieta control, especialmente en aquellas que incluían guisante Iceberg y Luna.

En el caso del Ensayo 2, hubo, igualmente, cuatro tratamientos pero con porcentajes crecientes del mismo ALB: 0\% (dieta control), $5 \%, 15 \%$ y $25 \%$; en consecuencia también se incrementó la cantidad de GEC. Se analizaron previamente tres variedades de ALB (ZV-220, ZV-274 e IC-2470) y finalmente se utilizó la que tenía menor contenido de GEC $(1,52 \%$ vs. $1,61 \%$ vs. $1,65 \%$, respectivamente) y que, por este motivo, se podría introducir en mayor porcentaje en el pienso. La harina de soja se redujo de forma proporcional (Tabla 3).

\section{Controles productivos}

En ambos ensayos se registró el peso individual al inicio (P0) y final del periodo (P1), uti- lizando una báscula modelo S-4C (Sipesa, Girona, España), así como el consumo medio diario (CMD) por celda (diferencia entre pienso añadido y consumido). Esos datos se utilizaron para calcular la ganancia media diaria (GMD) y la conversión alimenticia (IC) para cada réplica. No hubo problemas sanitarios durante las pruebas.

\section{Análisis de laboratorio}

La composición química de los piensos, guisantes y ALB fue determinada en el laboratorio de I+D Agroalimentario del ITACYL (finca Zamadueñas, Valladolid), y en el Departamento de Tecnología de los Alimentos, Sección de Tecnología Vegetal, del Instituto Nacional de Investigación y Tecnología Agraria y Alimentaria (INIA, Madrid y Soria), de acuerdo con los procedimientos descritos en el Diario Oficial de la Unión Europea (2009) (Tablas 1, 2 y 3: nutrientes y análisis determinados). La concentración de AA en guisantes se estimó mediante cromatografía de intercambio iónico (Hewlett-Packard 1100, Waldbronn, Alemania) después de hidrólisis ácida, aplicando el procedimiento descrito por Jones et al. (1981). Para valorar las concentraciones de metionina y cistina, muestras separadas se oxidaron con ácido perfórmico antes de la hidrólisis midiéndose como sulfona metionina y ácido cisteico, respectivamente (Moore, 1963). El triptófano se analizó mediante cromatografía líquida de alta resolución con fluorescencia, después de hidrólisis alcalina (hidróxido de bario) durante 20 horas a $110^{\circ} \mathrm{C}$ (Diario Oficial de la Unión Europea, 2009).

La actividad IT (UTI/mg de muestra; guisantes, soja y piensos), fue valorada con el método de Kakade et al. (1974) modificado por Grant et al. (1995) y Muzquiz et al. (2004). Para ello, se tomaron muestras por duplicado $(0,025 \mathrm{~g})$ que se molieron usando un molino (Retsch modelo Z-I, Sttutgart, Alemania) provisto de una criba de $0,5 \mathrm{~mm}$, para posteriormente ser ex- 
Tabla 2. Ingredientes y composición de los piensos del ensayo 1: CON (Control), P-CAR (Cartouche),

P-ICE (Iceberg), P-LUN (Luna) (en \% de materia fresca a no ser que se indique lo contrario)

Table 2. Ingredients and chemical composition of diets in trial 1: CON (Control), P-CAR (Cartouche), P-ICE (Iceberg), P-LUN (Luna) (\%, as-fed basis unless otherwise indicated)

\begin{tabular}{|c|c|c|c|c|}
\hline & CON & P-CAR & P-ICE & P-LUN \\
\hline \multicolumn{5}{|l|}{ Ingredientes } \\
\hline Guisante Cartouche & & 30,9 & & \\
\hline Guisante Iceberg & & & 54,1 & \\
\hline Guisante Luna & & & & 22,9 \\
\hline Cebada & 43,5 & 21 & 13 & 27,3 \\
\hline Trigo & 25 & 20 & 20 & 20 \\
\hline Harina de soja 47 & 16,2 & & & \\
\hline Soja extrusionada & 5 & 17,8 & 3 & 19,5 \\
\hline Concentrado y premix ${ }^{1}$ & 5 & 5 & 5 & 5 \\
\hline Grasa animal & 2,4 & 2 & 2,2 & 2 \\
\hline Fosfato dicálcico & 1,65 & 1,57 & 1,58 & 1,60 \\
\hline Carbonato cálcico & 0,45 & 1,06 & 0,59 & 1,03 \\
\hline Cloruro sódico & 0,20 & 0,20 & 0,20 & 0,20 \\
\hline L-Lisina 50\% & 0,49 & 0,26 & 0,12 & 0,31 \\
\hline DL-Metionina & 0,05 & 0,10 & 0,16 & 0,10 \\
\hline L-Treonina & 0,05 & 0,05 & 0,06 & 0,05 \\
\hline \multicolumn{5}{|l|}{ Análisis calculado } \\
\hline Energía neta (kcal/kg) & 2.414 & 2.433 & 2.402 & 2.445 \\
\hline Fibra bruta & 4,57 & 4,32 & 4,5 & 4,57 \\
\hline Lisina & 1,31 & 1,31 & 1,31 & 1,31 \\
\hline Metionina & 0,40 & 0,41 & 0,43 & 0,4 \\
\hline Metionina + Cistina & 0,74 & 0,75 & 0,75 & 0,74 \\
\hline Treonina & 0,80 & 0,80 & 0,81 & 0,80 \\
\hline Triptófano & 0,24 & 0,24 & 0,21 & 0,24 \\
\hline \multicolumn{5}{|l|}{ Análisis determinado } \\
\hline Materia seca & 90,5 & 90,0 & 90,5 & 90,2 \\
\hline Proteína bruta & 19,1 & 19,1 & 18,9 & 19,1 \\
\hline Lisina & 1,27 & 1,28 & 1,28 & 1,30 \\
\hline Extracto etéreo & 5,3 & 6,2 & 4,9 & 6,8 \\
\hline Almidón & 40,35 & 38,65 & 43,85 & 38,51 \\
\hline Cenizas & 6,28 & 6,12 & 6,00 & 6,07 \\
\hline UTI (unidades/mg)² & 0,97 & 1,62 & 3,09 & 3,19 \\
\hline UQI (unidades/mg) ${ }^{3}$ & 1,94 & 3,16 & 4,95 & 5,56 \\
\hline
\end{tabular}

${ }^{1}$ Ingredientes \%: concentrado de proteína de soja 62, 31; gluten de maíz 60, 30; proteína de maíz y trigo, 13; vitaminas y minerales, 8 ; harina de soja 47,5 ; hidrolizado de proteína porcina, 5; proteína de patata, 4; aceite de soja, 0,5; aminoácidos, 3,5. Composición química \%: concentrado de proteína, 4,6 (humedad, 10,16; cenizas, 11,58; proteína cruda, 70,5; grasa bruta, 5; fibra bruta, 2,61); Vitaminas y minerales, 0,4 (10.000 Ul vitamina A/kg, $2.000 \mathrm{UI}$ vitamina $\mathrm{D}^{3} / \mathrm{kg}, 20 \mathrm{mg}$ vitamina $\mathrm{E}$ ( $\alpha$-tocoferol)/ $/ \mathrm{kg}, 125 \mathrm{mg} / \mathrm{kg}$ de $\mathrm{Cu}$ (sulfato de cobre), $80 \mathrm{mg} / \mathrm{kg}$ de óxido de zinc. ${ }^{2}$ Inhibidores de tripsina. ${ }^{3}$ Inhibidores de quimotripsina. 
Tabla 3. Ingredientes y composición de los piensos experimentales del ensayo 2 (\%) Table 3. Ingredients and chemical composition of experimental diets in trial 2 (\%)

\begin{tabular}{|c|c|c|c|c|}
\hline & $0 \%$ & $5 \%$ & $15 \%$ & $25 \%$ \\
\hline \multicolumn{5}{|l|}{ Ingredientes } \\
\hline Alberjón & & 5 & 15 & 25 \\
\hline Cebada & 45,91 & 42,98 & 35,94 & 28,21 \\
\hline Trigo & 25 & 25 & 25 & 25 \\
\hline Harina de soja 47 & 12,50 & 10,70 & 8,20 & 6,20 \\
\hline Soja extrusionada & 5 & 5 & 5 & 5 \\
\hline Concentrado y premix ${ }^{1}$ & 5 & 5 & 5 & 5 \\
\hline Grasa animal & 3,2 & 3,1 & 3,1 & 3,2 \\
\hline Fosfato dicálcico & 1,63 & 1,60 & 1,52 & 1,44 \\
\hline L-Lisina 50\% & 0,67 & 0,55 & 0,24 & \\
\hline Carbonato cálcico & 0,62 & 0,64 & 0,68 & 0,72 \\
\hline Cloruro sódico & 0,20 & 0,20 & 0,20 & 0,20 \\
\hline DL-Metionina & 0,10 & 0,09 & 0,06 & 0,03 \\
\hline L-Treonina & 0,16 & 0,13 & 0,05 & \\
\hline \multicolumn{5}{|l|}{ Composición calculada } \\
\hline Energía neta (Kcal/kg) & 2.450 & 2.448 & 2.448 & 2.450 \\
\hline Fibra bruta & 3,73 & 4,10 & 4,82 & 5,54 \\
\hline Lisina & 1,25 & 1,24 & 1,24 & 1,29 \\
\hline Metionina & 0,40 & 0,40 & 0,39 & 0,38 \\
\hline Metionina + Cistina & 0,72 & 0,72 & 0,72 & 0,73 \\
\hline Treonina & 0,80 & 0,81 & 0,81 & 0,85 \\
\hline Triptófano & 0,23 & 0,23 & 0,22 & 0,23 \\
\hline \multicolumn{5}{|l|}{ Análisis determinado } \\
\hline Materia seca & 90,0 & 89,8 & 89,7 & 89,7 \\
\hline Proteína bruta & 17,9 & 18,1 & 18,0 & 18,32 \\
\hline Lisina & 1,22 & 1,25 & 1,24 & 1,28 \\
\hline Grasa bruta & 5,3 & 5,2 & 5,5 & 5,2 \\
\hline Almidón & 38,9 & 39 & 39,1 & 40,2 \\
\hline Cenizas & 6,5 & 5,5 & 5,4 & 5,8 \\
\hline
\end{tabular}

${ }^{1}$ Ingredientes \%: concentrado de proteína de soja 62, 31; gluten de maíz 60, 30; proteína de maíz y trigo, 13; vitaminas y minerales, 8 ; harina de soja 47,5 ; hidrolizado de proteína porcina, 5; proteína de patata, 4; aceite de soja, 0,5; aminoácidos, 3,5. Composición química \%: concentrado de proteína, 4,6 (humedad, 10,16; cenizas, 11,58; proteína cruda, 70,5; grasa bruta, 5; fibra bruta, 2,61); Vitaminas y minerales, 0,4 (10000 UI vitamina A $/ \mathrm{kg}, 2000 \mathrm{UI}$ vitamina D $3 / \mathrm{kg}, 20 \mathrm{mg}$ vitamina $\mathrm{E}$ ( $\alpha$-tocoferol)/kg, $125 \mathrm{mg} / \mathrm{kg}$ de $\mathrm{Cu}$ (sulfato de cobre), $80 \mathrm{mg} / \mathrm{kg}$ de óxido de zinc. 
traída con $1 \mathrm{ml}$ de $\mathrm{HCl}$ 0,05 molar, mediante un agitador orbital durante una hora, a una temperatura de $5^{\circ} \mathrm{C}$. El extracto se centrifugó a $11.000 \times g$ durante $4 \mathrm{~min}$, y el sobrenadante se midió para el cálculo de la actividad, usando $\mathrm{N}$ - $\alpha$-Benzoil-DL-Arginina-pNitroanilida como sustrato específico. La actividad IT se calculó por lectura de absorbancia contra un blanco a $410 \mathrm{~nm}$ usando un espectrofotómetro ultravioleta (modelo DU-7, Beckmann Instruments Inc., Fullerton, EE. UU.).

En el caso de la actividad IQ (UQI/mg muestra) se empleó el procedimiento de Sathe y Salunkhe (1981). Las muestras se procesaron del mismo modo que para la actividad IT y con una solución tampón (100 mM Tris- $\mathrm{HCl}$ conteniendo $20 \mathrm{mM}$ de $\mathrm{CaCl}_{2}$ y pH 7,8 ) a $30^{\circ} \mathrm{C}$ con N-Benzoil-L-Tirosina-Etil Ester como sustrato. La absorbancia se midió 7 minutos después de la adición del sustrato a $256 \mathrm{~nm}$ con el equipo indicado para la actividad IT.

El contenido de GEC del ALB se calculó siguiendo los procedimientos de Arias et al. (2004) y Sánchez-Vioque et al. (2011) por cromatografía líquida de alta resolución. El registro y tratamiento de los datos fueron llevados a cabo con el software 32 Karat versión 7.0 (Beckman-Coulter, Brea, CA, EE.UU.). Los extractos $(20 \mu \mathrm{l})$ se inyectaron directamente en una columna Discovery BIO Amplia Pore C18 (25 cm x 4,6 mm, 5 micras) (Supelco, Bellefonte, PA, EE.UU.).

Se determinó la concentración de otros FAN como inositoles fosfato totales, una vez extraídos con $\mathrm{HCl}$ y concentrados y purificados en una columna de intercambio aniónico, mediante cromatografía líquida de alta resolución en fase inversa (Beckman System Gold, EE.UU.), usando el método de Burbano et al. (1995). Los compuestos fenólicos se extrajeron mediante una solución de metanol y $\mathrm{HCl}$, siendo cuantificados según la capacidad de absorbancia de radicales oxígeno siguiendo el método de Dávalos et al., 2004. Los azúcares solubles se obtuvieron a partir de harina tratada con etanol diluido (50\%); el extracto se analizó mediante cromatografía líquida (Beckman System Gold, EE.UU.) con la técnica de Muzquiz et al. (1992). Las lectinas se estimaron por la actividad hemoaglutinante y valoradas con extractos salinos buffer fosfato mediante un procedimiento de dilución en serie utilizando células sanguíneas de rata tratadas con tripsina. La cantidad de material (mg) que causó $50 \%$ de eritrocitos aglutinados se definió como aquella que contenía 1 unidad hemoaglutinante (HU) (Grant, 1991).

\section{Análisis estadístico}

El diseño experimental fue en bloques completos al azar, utilizándose para analizar los datos los procedimientos GLM y REG del programa estadístico SAS (2004). En ambos experimentos, cada tratamiento se repitió ocho veces (dos en cada sala), considerando la celda con seis cerdos la réplica. Los resultados se muestran en tablas como medias corregidas por mínimos cuadrados.

El modelo fue: $Y_{i j}=\mu+$ Dieta $_{i}+\alpha P 0+\varepsilon_{i j}$; donde $Y$ : variables dependientes (GMD, CMD, IC, Peso final, ingesta media diaria de IT e IQ); $\mu$ : media poblacional; Dieta: nivel dietético de IP (ensayo 1) de alberjón y GEC (ensayo 2); $\alpha$ : coeficiente de regresión parcial entre peso inicial y variables dependientes, P0: peso inicial (utilizado como covariable) y $\varepsilon$ : error residual. Las medias se compararon mediante un test$t$, siendo $P<0,05$ clasificada como la diferencia significativa y $P<0,10$ tendencia.

También se obtuvieron ecuaciones de regresión múltiple, Ensayo 1, que relacionaron GMD, CMD e IC con la ingesta diaria de IT, IQ y la ratio IT/IQ, y la de alberjón y GEC en el ensayo 2. Igualmente, en este último se determinaron contrastes ortogonales comparando medias productivas según presencia y porcentaje de alberjón en la dieta. 


\section{Resultados y discusión}

\section{Análisis de materias primas proteicas $y$ dietas}

La composición química de los ingredientes proteicos (harina de soja, soja extrusionada, guisantes, alberjón) y las diferentes dietas se muestra en las Tablas 1, 2 y 3 . Los guisantes utilizados eran variedades de invierno y tenían un mayor contenido de PB que el reportado por FEDNA (2010) para guisante primavera (20,6\%). El perfil de aminoácidos, en general, fue similar para los tres guisantes con la excepción de algunos aminoácidos específicos: CAR tuvo el nivel más bajo de lisina e ICE el de valina. Además, CAR mostró la mayor proporción de extracto etéreo y la menor de fibra bruta.

Los FAN de guisantes que se encuentran en cantidad suficiente para provocar problemas son los IP (IT, IQ; Tabla 1), y suponen el factor limitante para incluirlos en cantidades elevadas en los piensos al disminuir la digestibilidad de la proteína (Le Gall et al., 2007). Según la cantidad de IT, el guisante ICE se considera de baja actividad, CAR de actividad media y LUN de alta actividad (Carrouée et al., 1994). Existen numerosos estudios que relacionan la actividad IP exclusivamente con los inhibidores de tripsina, sin información detallada de la relación con los inhibidores de quimotripsina, aunque la cantidad de estos últimos es mayor en los tres guisantes empleados, al igual que su capacidad inhibidora (Grosjean et al., 2000). Los resultados de la Tabla 2 muestran el análisis de IP en los piensos; aumentaron en relación con el pienso control, triplicando prácticamente los valores de ambos inhibidores (IT, IQ) en el pienso con LUN.

En la Tabla 1 también se observa la composición química del ALB utilizado en el Ensayo 2. El contenido de PB $(26,5 \%)$ fue similar a la encontrada por Wali et al. (2005) (25,8\%), pero ligeramente superior a la observada en otros estudios (Brand et al., 2004), probablemente debido a la variedad utilizada. El valor de lisina fue intermedio $(1,77 \%)$ si tenemos en cuenta que puede llegar al $2 \%$ según Hadjipanayiotou y Economides (2001). Además, los contenidos en metionina y en treonina fueron superiores a los presentados en las tres variedades de guisantes. Dichos AA son de interés para dietas de lechones según muestran las necesidades de nutrientes de FEDNA (2013). El caso de los AA azufrados, metionina + cistina, merece una consideración especial. La semilla de ALB tiene mayor contenido de azufre $(0,28$ a $0,37 \%)$ que otras legumbres generalmente incluidas en los piensos como el guisante $(0,18 \%)$ (FEDNA, 2010), y se debe, al menos en parte, al contenido de GEC, factor antinutritivo que tiene una presencia considerable de este elemento (11,6\%) (Enneking, 1995b). El azufre transmite sabores y olores desagradables al alimento, afecta a la palatabilidad y por lo tanto disminuye la ingesta de pienso (Arias-Royo et al., 2006; Tate y Enneking, 2006).

El contenido de extracto etéreo del ALB fue similar al detectado en guisante CAR, por el contrario, el almidón fue más alto en todas las variedades de guisantes (Tabla 1).

\section{Ensayo 1. Guisantes de invierno (IP)}

Los ensayos con guisantes en dietas para lechones son numerosos y difieren en sus resultados, ya sea por la edad de los animales, los niveles de IP, la aplicación de tratamientos previos, o debido a su bajo nivel de inclusión en las dietas. La Tabla 4 muestra la influencia de diferentes niveles de IP en el pienso estárter sobre el rendimiento de los lechones.

Los niveles de IP (IT, IQ) tolerados, sin mermas productivas, en P-LUN, coinciden con los descritos por Batterham et al. (1993): 4,7 UTI, 4,5 UQI, que compararon una dieta sin guisante, con garbanzos (Cicer arietinum) y guandú (Cajanus cajan) frente a un control de soja, pero con cerdos de 20 a $50 \mathrm{~kg}$ de peso, sin en- 
Tabla 4. Influencia del contenido en inhibidores de proteasas de las dietas: CON (Control),

P-CAR (guisante Cartouche), P-ICE (guisante Iceberg), P-LUN (guisante Luna), sobre el rendimiento productivo de lechones

Table 4. Influence of the dietary level of protease inhibitors: CON (Control), P-CAR (pea Cartouche), $P$-ICE (pea Iceberg), P-LUN (pea Luna), on performance traits of piglets

\begin{tabular}{|c|c|c|c|c|c|c|}
\hline Variable & CON & P-CAR & P-ICE & P-LUN & ESM $^{1}$ & $P^{2}$ \\
\hline Peso inicial, kg & 11,35 & 11,34 & 11,11 & 11,10 & \multicolumn{2}{|c|}{ Covariable } \\
\hline Peso final, kg & $21,92^{b}$ & $23,76^{a}$ & $21,47^{b}$ & $21,51^{b}$ & 0,33 & 0,001 \\
\hline Ganancia media diaria, g/d & $509^{b}$ & $590^{a}$ & $487^{b}$ & $490^{b}$ & 10 & 0,001 \\
\hline Consumo medio diario, g/d & 852 & 897 & 829 & 834 & 40 & 0,580 \\
\hline Índice de conversión, kg/kg & 1,69 & 1,51 & 1,70 & 1,69 & 0,06 & 0,100 \\
\hline Consumo de $\mathrm{IT}^{3}$ (106 unidades/día) & $0,79^{c}$ & $1,43^{b}$ & $2,56^{\mathrm{a}}$ & $2,67^{a}$ & 0,09 & 0,001 \\
\hline Consumo de IQ ${ }^{4}$ (10 ${ }^{6}$ unidades/día) & $1,60^{d}$ & $2,80^{c}$ & $4,11^{\mathrm{b}}$ & $4,65^{a}$ & 0,17 & 0,001 \\
\hline
\end{tabular}

${ }^{1}$ ESM: error estándar de la media $(n=8)$. ${ }^{2}$ Probabilidad; letras diferentes en la misma fila indican diferencias significativas. ${ }^{3}$ Inhibidores de tripsina. ${ }^{4}$ Inhibidores de quimotripsina.

contrar diferencias productivas $(P>0,05)$. Parece que no hay un efecto negativo, al menos hasta los niveles de IP que tiene el pienso PLUN. De hecho, los cerdos que consumieron P-CAR, con mayor cantidad de IP que los que consumieron CON, crecieron un $8,3 \%$ más.

En lechones de 6,5 a 17,7 kg de peso, Brooks et al. (2009) probaron una dieta con $20 \%$ de guisantes de primavera frente a una dieta control con maíz y harina de soja. Los cerdos que comieron el guisante mejoraron el crecimiento (418 vs. $385 \mathrm{~g}$ ), aumentaron el consumo de pienso (632 vs. $561 \mathrm{~g}$ ) y presentaron peor eficiencia alimentaria (660 vs. $685 \mathrm{~g} / \mathrm{kg}$ de pienso) de manera significativa $(P=0,01)$.

Se puede incrementar la proteína de la dieta utilizando concentrado de proteína de guisante (PB: $52,5 \%$ ) obtenido por micronizado. El problema es que se aumenta igualmente la cantidad de IP, como indican Valencia et al. (2008) en una prueba con lechones de 26 a 48 días alimentados con dos dietas, con 10,5\% de concentrado de proteína de guisante y con $12,1 \%$ de harina de soja (PB: $45,2 \%$ ); el con- sumo fue similar, aunque los primeros tuvieron peor rendimiento debido a la menor digestibilidad de la proteína $(P<0,05)$.

El procesado de guisantes con un bajo contenido de IP no afecta a los parámetros productivos. Grosjean et al. (1997) proporcionaron dos dieta con $40 \%$ de guisantes (2,4 UTI) crudos o extrusionados, a lechones de 27 a 68 días de edad; no hubo diferencias de consumo ni crecimiento en comparación con una dieta control de soja $(P>0,05)$. Por el contrario, en una segunda prueba de los mismos autores, la alimentación con un $40 \%$ de guisantes con altas cantidades de UTI $(10,4)$ frente a guisantes con 2 UTI, también en el mismo porcentaje, redujo la GMD (371 vs. $481 \mathrm{~g}$ ) y el CMD (670 vs. $726 \mathrm{~g})$ de manera significativa $(P>0,05)$. Stein y Peters (2008), en la fase de estárter obtuvieron resultados similares, con dietas a base de maíz y soja frente a una dieta con $60 \%$ de guisantes de primavera $(P>0,05)$; la extrusión previa no mejoró los resultados productivos en los lechones alimentados con los guisantes, lo que indica que se trataba de una variedad con bajos niveles de IP. 
La posible reducción del CMD, que refieren diferentes autores, puede ser debida a varias causas. McLaughlin et al. (1983) concluyeron que altos niveles de IT podrían desencadenar la liberación de colecistoquinina (CCK), hormona relacionada con la saciedad; para Grosjean et al. (1997) los IT reducirían la digestibilidad del triptófano, AA con efecto sobre el apetito. Jaikaran et al. (2002) en un ensayo con guisantes de diferentes variedades (amarillo y verde) y cerdos de más de 25 $\mathrm{kg}$, no encontraron diferencias en el CMD en comparación con una dieta control de soja. La presencia de saponinas en los guisantes, que según la variedad de guisante y el tipo de saponina (B o DDMP) varía entre 0 y 1,5 $\mathrm{g} / \mathrm{kg}$, podría reducir el consumo debido al sabor amargo (Heng et al., 2006), aunque Price et al. (1985) sugieren que el rechazo parcial de la dieta se manifiesta desde los 2 $\mathrm{g} / \mathrm{kg}$ de saponina.

El consumo medio de la dieta fue similar en los cuatro tratamientos, sin embargo los cerdos alimentados con P-CAR mejoraron el crecimiento y la conversión $(P<0,05)$; el motivo por el cual se observaron dichos resultados tiene difícil explicación, por lo que se debe seguir estudiando.

Teniendo en cuenta los resultados de la literatura, se esperaba una relación entre la ingesta diaria de inhibidores de proteína y los rendimientos, determinándose distintas ecuaciones de regresión (Tabla 5). En ellas, los niveles de IP y su relación (UTI/UQI) explicaron el $43,57,50$ y $28 \%$ de la variación del peso final, consumo, crecimiento e índice de conversión, respectivamente $(P<0,05)$.

Tabla 5. Ecuaciones de regresión entre los parámetros productivos y el consumo medio diario de inhibidores de tripsina (UTI) y quimotripsina (UQI) (unidades de inhibidor/mg de pienso diario $\times 10^{6}$ ) en lechones

Table 5. Regression equations between performance traits ( $A D F I, A D G, F C R$ ) and the average daily intake of trypsin (TI) and chymotrypsin (CI) inhibitors (inhibitor units/mg feed $\times 10^{6}$ ) in piglets

\begin{tabular}{|c|c|c|c|}
\hline Ecuaciones de regresión ${ }^{1}$ & $\mathrm{R}^{22}$ & $\mathrm{DRS}^{3}$ & $P^{4}$ \\
\hline$P F=64,662-(12,497 \cdot U T I)-(5,052 \cdot$ UQI $)-(88,987 \cdot(U T I / U Q I))$ & 0,43 & 2,30 & 0,001 \\
\hline $\mathrm{CMD}=3,954+(1,095 \cdot \mathrm{UTI})-(0.482 \cdot \mathrm{UQI})-(6,435 \cdot(\mathrm{UTI} / \mathrm{UQI}))$ & 0,57 & 0,11 & 0,0001 \\
\hline GMD $=1,148-(0,039 \cdot$ UTI $)+(0,083 \cdot$ UQI $)-(1,483 \cdot($ UTI/UQI $))$ & 0,50 & 0,06 & 0,0002 \\
\hline $\mathrm{IC}=5,887+(2,362 \cdot \mathrm{UTI})-(1,266 \cdot \mathrm{UQI})-(8,145 \cdot(\mathrm{UTI} / \mathrm{UQI}))$ & 0,28 & 0,16 & 0,024 \\
\hline
\end{tabular}

${ }^{1}$ Variables dependientes: peso final (PF, kg); ganancia media diaria (GMD, kg/día); consumo medio diario (CMD, kg/día); índice de conversión (IC, kg/kg). Variables independientes: unidades de tripsina inhibida (UTI: $10^{6}$ unidades/día); unidades de quimotripsina inhibida (UQI: $10^{6}$ unidades/día). ${ }^{2}{ }^{2}$ : coeficiente de determinación. ${ }^{3}$ DRS: desviación residual estándar. ${ }^{4}$ Probabilidad.

\section{Ensayo 2. Alberjón (GEC)}

La Tabla 6 muestra el efecto de los niveles de ALB sobre el crecimiento de los cerdos durante el período de estárter. Los animales alimentados con la dieta del $5 \%$ de ALB co- mieron y crecieron más $(P<0,001)$ que los de la dietas control y $25 \%$. El IC fue mejor con el control y el $5 \%$ que con el $25 \%$, estando en una posición intermedia los lechones alimentados con el pienso que contenía un $15 \%$ de ALB. Al final del periodo experi- 
Tabla 6. Influencia del porcentaje de alberjones en el pienso sobre el rendimiento productivo

Table 6. Influence of the dietary level of narbon vetch about performance traits of piglets

\begin{tabular}{lcccccc}
\hline Variable & $0 \%$ & $5 \%$ & $15 \%$ & $25 \%$ & ESM $^{1}$ & $P^{2}$ \\
\hline Peso inicial (kg) & 11,56 & 11,54 & 11,47 & 11,62 & \multicolumn{2}{c}{ Covariate } \\
Peso final (kg) & $21,20^{\mathrm{b}}$ & $23,40^{\mathrm{a}}$ & $20,73^{\mathrm{b}}$ & $18,28^{\mathrm{c}}$ & 0,365 & 0,001 \\
Ganancia media diaria (g/día) & $536^{\mathrm{b}}$ & $652^{\mathrm{a}}$ & $505^{\mathrm{b}}$ & $373^{\mathrm{c}}$ & 0,018 & 0,001 \\
Consumo medio diario (g/día) & $778^{\mathrm{b}}$ & $947^{\mathrm{a}}$ & $788^{\mathrm{b}}$ & $630^{\mathrm{c}}$ & 0,026 & 0,001 \\
Índice de conversión (kg/kg) & $1,45^{\mathrm{c}}$ & $1,45^{\mathrm{c}}$ & $1,56^{\mathrm{b}}$ & $1,68^{\mathrm{a}}$ & 0,028 & 0,001 \\
\hline
\end{tabular}

${ }^{1} \mathrm{ESM}$ : error estándar de la media $(n=8)$. ${ }^{2}$ Probabilidad; letras diferentes en la misma fila indican diferencias significativas.

mental, los cerdos alimentados con el $5 \%$ fueron los más pesados, y los que consumieron el $25 \%$ los más ligeros $(P<0,001)$.

Los contrastes ortogonales comparando el efecto de la presencia de ALB y sus proporciones se muestran en la Tabla 7.

Del mismo modo, la relación entre las variables productivas y la cantidad de ALB (\%) y el consumo de GEC (g) de la dieta se ajustaron a funciones cúbicas. La variable ALB explicó el 79, 60 y $54 \%$ de la variación de la GMD, CMD e IC respectivamente, siendo en el caso del GEC del 60, 44 y $67 \%$ para las mismas variables. Las ecuaciones obtenidas, que muestra la Tabla 8, proporcionaron información interesante bajo el punto de vista de la formulación de dietas para cerdos.

No hay demasiada información científica sobre el uso de ALB en lechones. La presencia de GEC parece ser determinante en las diferencias de consumo observado en el ensayo. De hecho, el CMD fue un 33,5\% menor en los cerdos que comieron un $25 \%$ de ALB en relación con los del $5 \%$.

Una estrategia para mejorar el consumo podría ser la eliminación del GEC después de la cosecha. Enneking (1995b) trabajó con tres dietas: $35 \%$ guisantes, $35 \%$ de ALB tratado previamente durante $12 \mathrm{~h}$ con una solución de ácido acético al $4 \%$ a $121^{\circ} \mathrm{C} 30$ min en un autoclave, y $35 \%$ de ALB tratado solo en autoclave, y no encontró ninguna diferencia en la GMD entre las dos primeras dietas. Una explicación podría ser que el ácido disminuyó la actividad del GEC, pero al ser termorresistente el tratamiento sólo con autoclave no fue eficaz.

La variedad de ALB utilizada en el ensayo no fue tratada con anterioridad, sólo se molió, mezcló y granuló con el resto de los ingredientes. En consecuencia, mayores cantidades de alberjones en la dieta originaron niveles más elevados de GEC, penalizando el consumo y el crecimiento. Por lo tanto, es importante la selección de variedades de ALB con bajo contenido de GEC. EI ALB utilizado mostró un nivel medio-bajo de este FAN, si consideramos las cantidades que refleja la literatura y que oscilan del 0,4 al 3,8\% (Enneking et al., 1998). Sin embargo, hay que tener en cuenta, igualmente, que el contenido de GEC depende, al menos en parte, de la variedad de semilla, las condiciones de cultivo, y la composición de azufre de suelo (Enneking y Wink, 2000; Arias-Royo et al., 2006). 
Tabla 7. Ensayo 2. Contrastes ortogonales entre las distintas variables productivas (P-valor) Table 7. Trial 2. Orthogonal contrasts of the different production variables (P-value)

\begin{tabular}{llllll}
\hline Contrastes & & $\mathrm{PF}^{1}$ & $\mathrm{CMD}^{2}$ & $\mathrm{GMD}^{3}$ & $\mathrm{IC}^{4}$ \\
\cline { 1 - 3 } \cline { 4 - 6 } $0 \%$ vs. $5 \%+15 \%+25 \%$ & & 0,345 & 0,736 & 0,231 & 0,0024 \\
$5 \%$ vs. $15 \%+25 \%$ & 0,0001 & 0,0001 & 0,0001 & 0,0001 \\
$15 \%$ vs. $25 \%$ & 0,0001 & 0,0004 & 0,0001 & 0,0072 \\
\hline
\end{tabular}

${ }^{1} \mathrm{PF}$ : peso final, kg. ${ }^{2} \mathrm{CMD}$, consumo medio diario (kg/día). ${ }^{3} \mathrm{GMD}$, ganancia media diaria (kg/día).

${ }^{4} \mathrm{IC}$, índice de conversión $(\mathrm{kg} / \mathrm{kg})$.

Tabla 8. Ensayo 2. Ecuaciones de regresión entre los parámetros productivos y el consumo medio diario de alberjones (0-25\%) y de GEC (g) en lechones Table 8. Trial 2. Regression equations between performance traits and the average daily intake of narbon vetch (0-25\%) and GEC (g) in piglets

\begin{tabular}{llll}
\hline Ecuaciones de regresión ${ }^{1}$ & $\mathrm{R}^{22}$ & $\mathrm{DRS}^{3}$ & $P^{4}$ \\
\hline $\mathrm{GMD}=0,536+0,043 \cdot \mathrm{VN}-0,0046 \cdot \mathrm{VN}^{2}+0,000103 \cdot \mathrm{VN}^{3}$ & 0,79 & 0,054 & 0,001 \\
$\mathrm{CMD}=0,778+0,060 \cdot \mathrm{VN}-0,0059 \cdot \mathrm{VN}^{2}+0,00013 \cdot \mathrm{VN}^{3}$ & 0,60 & 0,096 & 0,001 \\
$\mathrm{IC}=1,456-0,0074 \cdot \mathrm{VN}+0,0013 \cdot \mathrm{VN}^{2}-0,0000258 \cdot \mathrm{VN}^{3}$ & 0,54 & 0,096 & 0,001 \\
$\mathrm{GMD}=0,537+0,3618 \cdot \mathrm{GEC}-0,3534 \cdot \mathrm{GEC}^{2}+0,0737 \cdot \mathrm{GEC}^{3}$ & 0,60 & 0,076 & 0,001 \\
$\mathrm{CMD}=0,778+0,5214 \cdot \mathrm{GEC}-0,5021 \cdot \mathrm{GEC}+0,110 \cdot \mathrm{GEC}^{3}$ & 0,44 & 0,114 & 0,001 \\
$\mathrm{IC}=1,45-0,028 \cdot \mathrm{GEC}+0,0368 \cdot \mathrm{GEC}^{2}+0,0591 \cdot \mathrm{GEC}^{3}$ & 0,67 & 0,082 & 0,01 \\
\hline
\end{tabular}

${ }^{1} \mathrm{GMD}$, ganancia media diaria (kg/día); CMD, consumo medio diario kg/día); IC, índice de conversión

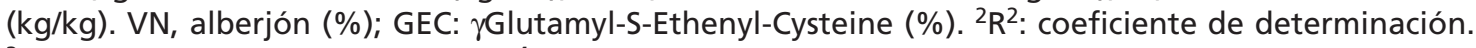
${ }^{3}$ DRS: desviación residual estándar. ${ }^{4} \mathrm{P}$ : probabilidad.

\section{Conclusiones}

En las condiciones experimentales en las que se han realizado ambos ensayos, se puede concluir que los lechones en la fase de estárter, mejoran o mantienen el rendimiento con ingestas diarias de al menos 2,67 y 4,65 unidades de tripsina y de quimotripsina inhibidas $\left(x 10^{6}\right)$, respectivamente, con tasas de $54,1 \%$ de guisantes en la dieta de acuerdo con la va- riedad, disminuyendo el porcentaje de soja de manera significativa.

La cantidad de GEC en el alberjón determina su inclusión en el pienso; la adición a la dieta de $5 \%$ de alberjón con un máximo de 1,52\% de GEC aumenta el crecimiento y el consumo significativamente. Porcentajes más altos del $15 \%$ empeoran la palatabilidad del pienso $y$, en consecuencia, el rendimiento. 


\section{Agradecimientos}

Este trabajo ha sido financiado por el Centro para el Desarrollo Tecnológico Industrial (Proyecto IDI-2010-0284) y por la Agencia de Inversiones y Servicios de Castilla y León (04-09-SO0013). Queremos agradecer al Dr. Marcelino De los Mozos (Centro de Investigación Agraria de Albaladejito, Cuenca) el apoyo en los análisis del GEC de los alberjones y a la Dra. Ana de Coca (Unión Regional de Cooperativas Agrarias de Castilla y León, Valladolid) la coordinación de los distintos grupos de investigación.

\section{Bibliografía}

Adamidou S, Nengas I, Grigorakis K, Nikolopoulou D, Jauncey K (2011). Chemical Composition and Antinutritional Factors of Field Peas (Pisum sativum), chickpeas (Cicer arietinum), and Faba Beans (Vicia faba) as affected by extrusion preconditioning and drying temperatures. Cereal Chemistry 88: 80-86.

Arias M, Ortiz LT, de los Mozos M (2004). Phenolic compounds and pyrimidine glycoside determination in Vicia narbonensis seed. En: Recent advances of research in antinutritional factors in legume seeds and oilseeds. Fourth International Workshop on antinutritional factors in legume seeds and oilseeds, 8-10 marzo, Toledo, Spain. EAAP publication $n^{\circ} 110$, pp. 29-33.

Arias-Royo M, Tate M, Enneking D (2006). Narbon bean (Vicia narbonensis L.): farmer's dream or devil's bean? Grain Legumes 47 (3rd quarter 2006): 18-19.

Batterham ES, Saini HS, Andersen LM, Baigent RD (1993). Tolerance of growing pigs to trypsin and chymotrypsin inhibitors in chickpeas (Cicer arietinum) and pigeonpeas (Cajanus cajan). Journal of Science Food and Agriculture 61: 211-216.

Bengala-Freire J, Hulin JC, Peiniau J, Aumaitre A (1989). Effet de la cuisson-extrusion du pois de printemps sur la digestibilité des aliments de sevrage precoce du porcelet et consequences sur les performances jusqu a I abattage. $21^{\text {es }}$ Jour- nées de la Recherche Porcine, 31-2 febrero, París, Francia, pp. 75-82.

BOE (2013). Real Decreto 53/2013, de 1 de febrero, por el que se establecen las normas básicas aplicables para la protección de los animales utilizados en experimentación y otros fines científicos, incluyendo la docencia. Boletín Oficial del Estado, núm 34, de 8 de febrero de 2013, pp. 11370-11421.

Brand TS, Brandt DA, Cruywagen CW (2004). Chemical composition, true metabolisable energy content and amino acid availability of grain legumes for poultry. South African Journal of Animal Science 34 (2): 116-122

Brooks KR, Wiegand BR, Meteer AL, Petersen GI, Spencer JD, Winter JR, Robb JAP (2009). Inclusion of yellow field peas and carbohydrase enzyme in nursery pig diets to improve growth performance. The Professional Animal Scientist 25: 17-25.

Burbano C, Cuadrado C, Muzquiz M, Cubero JI (1995). Variation of favism-inducing factors (vicine, convicine and L-DOPA) during pod development in Vicia faba L. Plant Foods for Human Nutrition 47: 265-274.

Carrouée B, Grosjean F, Peyronnet C, Weiss P (1994). Guisantes: utilización en Alimentación Animal. Union nationale interprofessionnelle des plantes riches en protéines, Institut technique des céréales et des fourrages, París. 96 pp.

Castleman GH (2000). A guide for feeding narbon beans to animals. Department of Natural Resources and Environment. Victorian Institute for Dryland Agriculture, Australia. 4 pp.

Crevieu-Gabriel I (1999). Digestion des protéines végétales chez les monogastriques. Exemple des protéines de pois. INRA Productions Animales 12(2): 147-161.

Dávalos A, Gómez-Cordovés C, Bartolomé B (2004). Extending applicability of the oxygen radical absorbance capacity (ORAC-fluorescein) assay. Journal Agriculture Food Chemistry 51: 48-54.

Diario Oficial de la Unión Europea (2009). Reglamento (CE) n 152/2009 de la Comisión de 27 de enero de 2009, por el que se establecen los mé- 
todos de muestreo y análisis para el control oficial de los piensos. Diario Oficial de la Unión Europea, L 54, de 26 de febrero de 2009, pp. 1-130.

Doti S, Suárez-Belloch J, Latorre MA, Guada JA, Fondevila M (2014). Effect of dietary starch source on growth performances, digestibility and quality traits of growing pigs. Livestock Science 164: 119-127.

Enneking D (1995a). Post-harvest detoxification: the key to alternative Vicia grain legumes? En: Lathyrus sativus and Human Lathyrism: Progress and Prospects. (Eds. Yusuf HKM, Lambein F), pp. 85-92. University of Dhaka. Dhaka (Bangladesh).

Enneking D (1995b). The toxicity of Vicia species and their utilisation as grain legumes. Centre for Legumes in Mediterranean Agriculture (CLIMA). Occasional Publication $\mathrm{N}^{\circ} 6$. University of Western Australia. Nedlands W.A. (First edition, Enneking, D. (1994) PhD thesis, University of Adelaide).

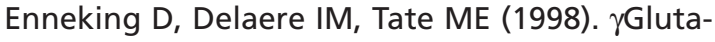
myl-S-Ethenyl-Cysteine: a dipeptide from Vicia narbonensis. Phytochemistry 48(4): 643-645.

Enneking D, Wink M (2000). Towards the elimination of antinutritional factors in grain legumes. En: Linking Research and Marketing Opportunities for Pulses in the 21st Century. Proceedings of the Third International Food Legume Research Conference (Ed. Knight R) pp. 375-384. Current Plant Science and Biotechnology in Agriculture Vol. 34. Kluwer Academic Publishers. Dordrecht/Boston/London.

FEDNA (2010). Tablas FEDNA de composición y valor nutritivo de alimentos para la fabricación de piensos compuestos. $3^{\mathrm{a}}$ Edición (Coordinada y Dirigida por C. de Blas, G. G. Mateos y P. García Rebollar). Ed. Fundación Española para el Desarrollo de la Nutrición Animal, Madrid. 502 pp.

FEDNA (2013). Necesidades nutricionales para ganado porcino: Normas FEDNA (Coordinada y Dirigida por C. de Blas, J. Gasa, G. G. Mateos). Ed. Fundación Española para el Desarrollo de la Nutrición Animal. Madrid. 114 pp.

García CA, Barrios A, Martín A, Rodríguez MJ, Caminero C (2006). La posible recuperación de algunas leguminosas (Vicia narbonensis L. y Lathy- rus cicera L.) para su cultivo en los secanos de Castilla y León. En: Nuevos Retos y Oportunidades de las Leguminosas en el Sector Agroalimentario Español. Segundas Jornadas de la Asociación Española de Leguminosa. 25-27 de abril, Cuenca, España. pp. 73-80. Consejería de Agricultura, Junta de Comunidades de Castilla-La Mancha.

González-García MR (2001). Interacción genotipo $x$ ambiente en guisante proteaginoso (Pisum sativum L.). Tesis Doctoral. Universidad de Valladolid.

Grant G (1991). Lectins. En: Toxic substances in crop plants (Eds. D'Mello JPF, Duffus C, Duffus JH), pp: 49-67. Royal Society Chemistry, Cambridge.

Grant G, Dorward PM, Buchan WC, Armour JC, Pusztai A (1995). Consumption of diets containing raw soya beans (Glycine max L.), kidney beans (Phaseolus vulgaris L.), cowpeas (Vigna unguiculata L.) or lupin seeds (Lupinus angustifolius L.) by rats for up to 700 days: Effects on body composition and organ weights. British Journal of Nutrition 73(1): 17-29.

Griffiths DW (1984). The trypsin and chymotrypsin inhibitor activities of various pea (Pisum spp.) and field bean (Vicia faba) cultivars. Journal of the Science of Food and Agriculture 35: 481-486.

Grosjean F, Jondreville C, Bogaert C, Bourdillon A, Peyronnet C, le Guen MP, Williatte I (1997). Utilisation d'aliments pour porcelets sevrés contenant $40 \%$ de pois. 29 es Journées de la Recherche Porcine, 4-6 febrero, París, Francia, pp. 197-204.

Grosjean F, Jondreville C, Williatte-Hazouard I, Skiba F, Carrouée B, Gâtel F (2000). Ileal digestibility of protein and amino acids of feed peas with different trypsin inhibitor activity in pigs. Canadian Journal of Animal Science 80: 643-652.

Guillamón E, Mateo-Vivaracho L, Villares A, D'Arrigo M, Pedrosa MM, García-Lafuente A, Muzquiz M (2012). Inhibidores de tripsina y de quimotripsina en semillas de leguminosas. IV Jornadas de la Asociación Española de leguminosas. 6-7 Junio, Pontevedra, España.

Hadjipanayiotou M, Economides S (2001). Chemical composition, in situ degradability and amino acid composition of protein supplements fed to livestock and poultry in Cyprus. Livestock Research for Rural Development 13(6). 
Heng L, Vincken JP, van Koningsveld GA, Legger A, Gruppen H, van Boekel MAJS, Roozen J, Voragen AGJ (2006). Bitterness of saponins and their content in dry peas. Journal of the Science of Food and Agriculture 86(8): 1225-1231.

Jaikaran S, Colangelo M, William C (2002). Growth and feed intake of growing pigs fed diets containing three varieties of field peas. Advances in Pork Production 13: 25.

Jones BN, Päävo S, Stein S (1981). Amino acids analysis and enzymatic sequence determination of peptides by an improved o-phthaldialdehyde precolumn labeling rocedure. Journal of Liquid Chromatography 4: 565-586.

Kakade ML, Rackis JJ, McGhee JE, Puski G (1974). Determination of trypsin inhibitor activity of soy products: a collaborative analysis of an improved procedure. Cereal Chemistry 51: 376-382.

Le Gall M, Quillien L, Sève B, Guéguen J, Lallès JP (2007). Weaned piglets display low gastrointestinal digestion of pea (Pisum sativum L.) lectin and pea albumin 2. Journal of Animal Science 85(11): 2972-2981.

Mateos GG, Valencia DG, Serrano MP, Lázaro R (2008). Las leguminosas de grano en alimentación animal: estudio del guisante. III Jornadas de la Asociación Española de Leguminosas. 2-4 septiembre, Valladolid, España.

McLaughlin CL, Peikin SR, Baile CA (1983). Trypsin inhibitor effects on food intake and weight gain in Zucker rats. Physiology and Behavior 31(4): 487-91.

Moore S (1963). On determination of cystine as cisteic acid. Journal of Biological Chemistry 238: 235-237.

Muzquiz M, Rey C, Cuadrado C, Fenwick R (1992). Effect of germination on oligosaccharide content of lupin species. Journal of Chromatography A 607: 349-352.

Muzquiz M, Hill GD, Cuadrado C, Pedrosa MM, Burbano C (2004). Recent advances of research in antinutritional factors in legume seeds and oilseeds. Proceedings of the fourth international workshop on antinutritional factors in legume seeds and oilseeds. EAAP Scientific Series, Volume 110. Wageningen, The Netherlans. 384 pp.
Muzquiz M (2012). Componentes nutricionalmente activos en leguminosas: implicaciones en nutrición y salud. IV Jornadas de la Asociación Española de Leguminosas. 6 y 7 de junio, Pontevedra, España.

Pisulewska E, Pisulewski PM (2000). Trypsin inhibitor activity of legume seeds (peas, chickling vetch, lentils, and soya beans) as affected by the technique of harvest. Animal Feed Science and Technology 86: 261-265.

Price KR, Griffiths NM, Curl CL, Fenwick GR (1985). Undiserable sensory properties of the dried pea (Pisum sativum). The role of saponina. Food Chemistry 17: 105-115.

Sánchez-Vioque R, Girón-Calle J, Rodriguez-Conde MF, Vioque J, De-los-Mozos-Pascual M, Santana-Méridas O, Izquierdo-Melero ME, Alaiz M (2011). Determination of $\gamma$-glutamyl-S-ethenylcysteine in narbon vetch (Vicia narbonensis L) seeds by high performance liquid Chromatography. Animal Feed Science and Technology 165: 125-130.

SAS (2004). User's Guide. SAS Institute. Cary, NC.

Sathe SK, Salunkhe DK (1981). Studies on trypsin and chymotrypsin inhibitory activities, hemagglutinating activity, and sugars in the Great Northern beans (Phaseolus vulgaris L.). Journal of Food Science 46: 626-629.

Stein H, Peters DN (2008). Effects of including field peas in diets fed to weanling pigs. Journal of Animal Science 86 (E-Suppl, 2): 448.

Tate M, Enneking D (2006). Vetches: from feed to food. Grain Legumes 47 (3rd quarter 2006).

Valencia DG, Serrano MP, Centeno C, Lázaro R, Mateos GG (2008). Pea protein as a substitute of soya bean protein in diets for Young pigs: Effects on productivity and digestive traits. Livestock Science 118: 1-10.

Wali SA, Hobi AA, Nouri A (2005). Some physiological and histological changes in the broilers fed gagon seeds (Narbon vetch). Journal of Biological Sciences 5(2): 111-113.

(Aceptado para publicación el 25 de abril de 2018) 